\title{
Involvement of circHIPK3 in the pathogenesis of diabetic cardiomyopathy in mice
}

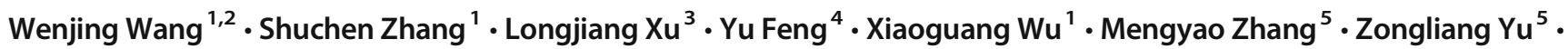 \\ Xiang Zhou ${ }^{1}$
}

Received: 28 August 2020 / Accepted: 12 November 2020 / Published online: 4 January 2021

(C) The Author(s), under exclusive licence to Springer-Verlag GmbH, DE part of Springer Nature 2021

\begin{abstract}
Aims/hypothesis In a mouse model of diabetic cardiomyopathy (DCM) the expression of the circular RNA circHIPK3 was found to be significantly increased. This study aimed to discover the molecular mechanisms linking circHIPK3 to the pathogenesis of DCM.

Methods The diabetic mouse model was established by i.p. injection of streptozotocin, which led to the development of DCM. Echocardiographic measurements were used to evaluate cardiac structure and function, and histological staining was applied to detect myocardial fibrosis in mice. 5-Ethynyl-2'-deoxyuridine incorporation was performed to determine cell proliferation and RNA fluorescent in situ hybridisation was employed to examine circHIPK3 expression in cardiac fibroblasts. RNA immunoprecipitation and luciferase reporter assay were conducted to explore the pathological mechanism of circHIPK3 in myocardial fibrosis.

Results Knockdown of circHIPK3 was found to attenuate myocardial fibrosis and enhance cardiac function in DCM mice. In addition, silencing of circHIPK 3 could suppress proliferation of cardiac fibroblasts treated with angiotensin II. Furthermore, RNA immunoprecipitation and luciferase reporter assay revealed a circHIPK3-miR-29b-3p-Colla 1-Col3al regulatory network in the pathogenesis of myocardial fibrosis.

Conclusions/interpretation circHIPK3 contributes to increased myocardial fibrosis during DCM by functioning as a competing endogenous RNA that upregulates Collal and Col3a1 expression through suppressing miR-29b-3p.
\end{abstract}

Keywords circHIPK $3 \cdot$ Diabetic cardiomyopathy $\cdot$ Myocardial fibrosis

\author{
Abbreviations \\ AAV-9 Adeno-associated virus 9 \\ AGO2 Argonaute 2 \\ Ang II Angiotensin II \\ ceRNA Competing endogenous RNA \\ circRNA Circular RNA \\ COL1A1 Collagen, type I, alpha 1
}
COL3A1 Collagen, type III, alpha 1
DCM Diabetic cardiomyopathy
FISH Fluorescent in situ hybridisation
LVEDD Left ventricular end-diastolic diameter
LVEF Left ventricular fractional ejection fraction
LVESD Left ventricular end-systolic diameter
LVFS Left ventricular fractional shortening fraction

Wenjing Wang, Shuchen Zhang and Longjiang Xu contributed equally to this work.

Xiang Zhou

zhou-xiang@suda.edu.cn

1 Department of Cardiology, The Second Affiliated Hospital of Soochow University, Suzhou, China

2 Department of Emergency, The Second Affiliated Hospital of Soochow University, Suzhou, China
3 Department of Pathology, The Second Affiliated Hospital of Soochow University, Suzhou, China

4 Department of Endocrinology, The Second Affiliated Hospital of Soochow University, Suzhou, China

5 Department of Cardiology, Kunshan First People's Hospital Affiliated to Jiangsu University, Kunshan, China 


\section{Research in context}

\section{What is already known about this subject?}

- Myocardial fibrosis is an important pathological change occurring in diabetic cardiomyopathy

- circHIPK3 is an abundant circular RNA involved in tumorigenesis and metabolic dysregulation

\section{What is the key question?}

- What is the molecular mechanism underlying the involvement of circHIPK3 in regulating diabetic cardiomyopathy?

What are the new findings?

- $\quad$ circHIPK3 knockdown can attenuate myocardial fibrosis and improve cardiac function in diabetic mice

- circHIPK3 silencing can inhibit proliferation of cardiac fibroblasts treated with angiotensin II

- $\quad$ The circHIPK3-miR-29b-3p-Col1a1-Col3a1 regulatory network is involved in the pathogenesis of diabetic myocardial fibrosis

How might this impact on clinical practice in the foreseeable future?

- circHIPK3 may become a potential therapeutic target for diabetic cardiomyopathy

miRNA MicroRNA

RIP RNA immunoprecipitation

shRNA Short hairpin RNA

STZ Streptozotocin developed a mouse model of DCM and found that circHIPK3 expression was significantly increased in the diabetic myocardium. We then explored the molecular mechanisms by which circHIPK 3 contributes to the development of DCM and found that circHIPK3 promotes myocardial fibrosis in DCM by inhibiting the miRNA miR-29b-3p.

\section{Introduction}

Diabetic cardiomyopathy (DCM) is an important complication of diabetes, manifesting mainly as ventricular remodelling, myocardial fibrosis and cardiac dysfunction. Emerging evidence shows that mitochondrial dysfunction, impaired calcium handling, oxidative stress, abnormalities in myocardial metabolism and diabetic microangiopathy are involved in the progression of DCM $[1,2]$. Myocardial fibrosis, a critical pathological change in DCM, increases myocardial stiffness, impairs cardiac function and provides the structural basis for ventricular arrhythmia [3]. However, the molecular mechanisms underlying myocardial fibrosis in the development of DCM need to be elucidated.

Circular RNAs (circRNAs) belong to a novel family of non-coding RNAs that are characterised by covalently closed continuous loops with no $5^{\prime}$ to $3^{\prime}$ polarity or polyadenylated tail [4]. circRNAs regulate gene expression by acting as RNAbinding protein sequestering agents, microRNA (miRNA) sponges and nuclear transcriptional regulators [5]. It has been shown that circRNAs regulate many pathways underlying CVDs [6, 7]. circHIPK3 is an abundant circRNA in tumorigenesis and metabolic dysregulation [8-10]. We have now

\section{Methods}

Animal model and treatment This study was approved by the Animal Ethics Committee of Soochow University and performed in compliance with the Animal Welfare Regulation in China. Male C57BL/6 mice, aged 8 weeks old, received streptozotocin $(\mathrm{STZ}, 50 \mathrm{mg} / \mathrm{kg}$ ) or vehicle (citrate buffer) by i.p. injection for 5 consecutive days. One week later, we measured fasting blood glucose levels with a glucometer and considered mice with glucose levels $>16.7 \mathrm{mmol} / \mathrm{l}$ to be diabetic. The diabetic mice were then randomly divided into three groups. One group received $2.5 \times 10^{10}$ viral genomes (VG) of adeno-associated virus 9 (AAV-9) incorporating circHIPK3-short hairpin RNA (shRNA) (DM + circHIPK3-shRNA) and a second group received an equal amount of AAV-9 incorporating scrambled-shRNA (DM + Scr-shRNA), both by intramyocardial injection at five locations through an open chest incision. A third group of diabetic mice received no treatment (DM), and we used non-diabetic mice as a control group. The mice were housed in the Experimental Animal Center of Soochow University for 12 weeks. The animal 
weight, blood glucose and blood pressure in each group are shown in electronic supplementary material (ESM) Table 1.

Cardiac fibroblast culture Neonatal mice were anaesthetised with isoflurane and the hearts were surgically removed. Ventricular tissues were cut into small pieces and digested in D-Hanks solution containing pancreatin and collagenase. We incubated the cell suspension in a culture flask for $90 \mathrm{~min}$ at $37^{\circ} \mathrm{C}$ to allow the fibroblasts to adhere preferentially to the flask bottom. We then removed non-adherent and weakly attached cells and incubated the cardiac fibroblasts with DMEM supplemented with L-glutamine, 10\% (vol./vol.) FBS and antibiotics $(100 \mathrm{U} / \mathrm{ml}$ penicillin and $100 \mathrm{mg} / \mathrm{ml}$ streptomycin) at $37^{\circ} \mathrm{C}$ in a humidified atmosphere. In in vitro experiments, cardiac fibroblasts were incubated with $10 \mu \mathrm{mol} / \mathrm{l}$ angiotensin II (Ang II) for $24 \mathrm{~h}$ to induce the fibrotic phenotype.

Echocardiographic assessment Twelve weeks after STZ injection, echocardiography was performed to assess cardiac structure and function in mice. The following measurements were obtained: left ventricular end-systolic diameter and enddiastolic diameter (LVESD, LVEDD), left ventricular fractional shortening and ejection fraction (LVFS, LVEF). All measurements were carried out by an experienced technician and averaged for three consecutive cardiac cycles.

Histological analysis and immunohistochemical staining Mouse myocardial tissue was surgically removed, fixed in $10 \%$ (vol./vol.) formalin, embedded in paraffin and sliced into sections (5 $\mu \mathrm{m}$ thick). The sections were stained with Masson's trichrome and H\&E. Digital images were collected from ten random fields of each section. To evaluate myocardial fibrosis and cardiac hypertrophy, collagen area fraction and myocyte cross-sectional area were measured using ImagePro Plus software. The operator was blinded to the group allocation. The expression of types I and III collagen was detected by immunohistochemistry. After antigen retrieval and quenching of endogenous peroxidase, we incubated the sections with $10 \%$ (vol./vol.) goat serum for $0.5 \mathrm{~h}$ at room temperature to block non-specific binding sites. We then incubated sections with rabbit anti-mouse collagen I and collagen III antibodies (Abcam, Cambridge, MA, USA; 1:200 in 3\% BSAT) at $4^{\circ} \mathrm{C}$ overnight. After washing with PBS, we incubated the slides with horseradish peroxidase-conjugated goat anti-rabbit secondary antibody for $0.5 \mathrm{~h}$ at room temperature.
Fig. 1 circHIPK3 expression pattern in mouse cardiac fibroblasts. (a) Real-time PCR was conducted to detect the expression of circHIPK3 and Hipk3 mRNA in cardiac fibroblasts after actinomycin D treatment (fold relative to $0 \mathrm{~h} ; n=$ $5,{ }^{*} p<0.05$ vs circHIPK3). (b) Total RNA was digested with RNase R, and then circHIPK3 and Hipk3 mRNA were examined by real-time PCR (fold relative to mock; $n=5, * p<0.05$ vs mock). (c) The expression of circHIPK3 and Hipk3 mRNA was determined by real-time PCR in the nuclear and cytoplasmic fractions of cardiac fibroblasts. (d) The expression of circHIPK3 was increased in cardiac fibroblasts exposed to high glucose $(30 \mathrm{mmol} / \mathrm{l})$ for $48 \mathrm{~h}$ (fold relative to control; $n=5$, $* p<0.05$ vs control). (e) Gel electrophoresis confirmed the PCR product of circHIPK 3 and Sanger sequencing verified the head-to-tail splicing of the product
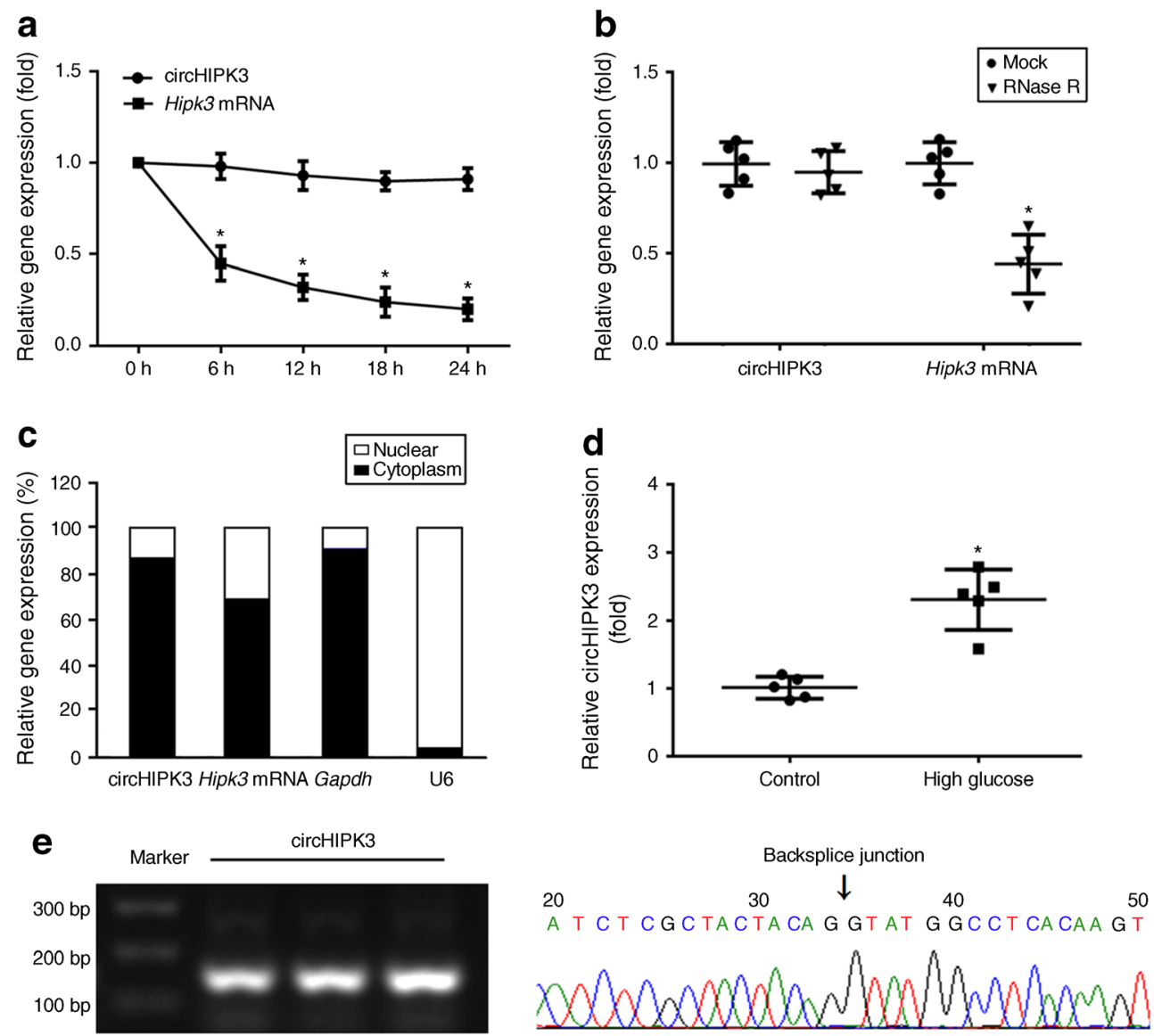

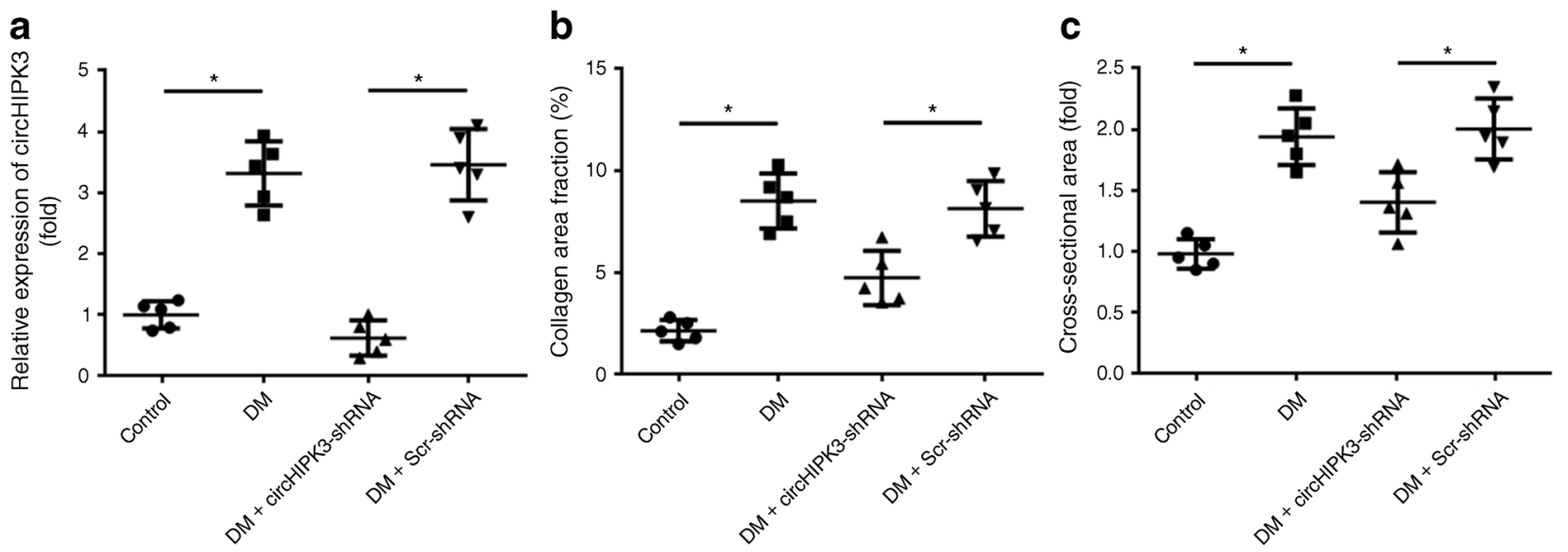
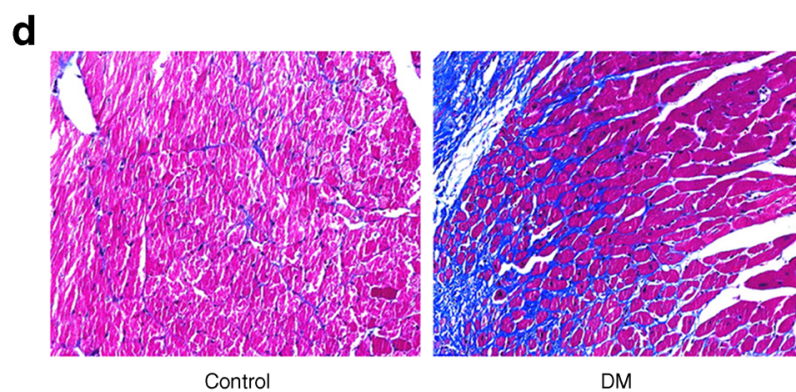

DM

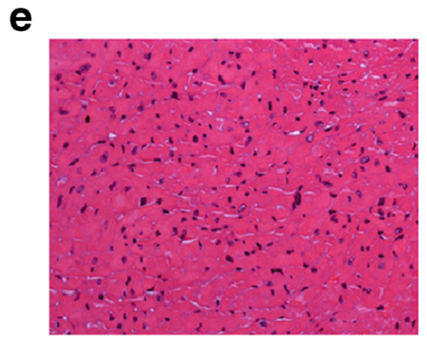

Control

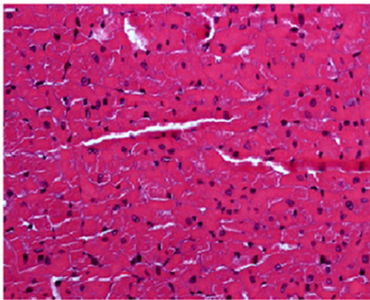

DM

Fig. 2 circHIPK3 silencing reduces histological abnormalities in diabetic mice. (a) The expression of circHIPK3 in myocardium was determined by real-time PCR. (b, d) Cardiac tissue was stained with Masson's trichrome and collagen area fraction was measured. $(\mathbf{c}, \mathbf{e})$ Cardiac tissue
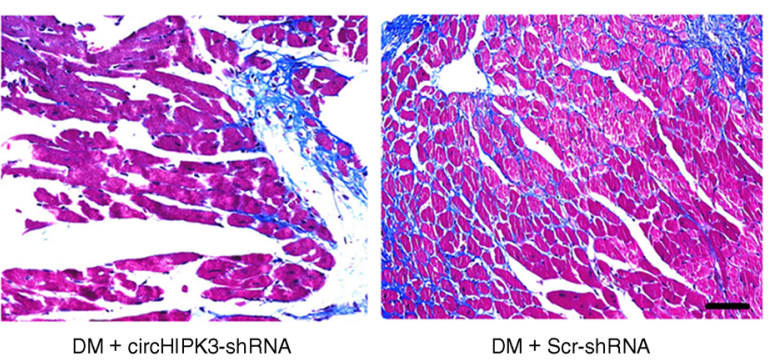

$D M+$ Scr-shRNA

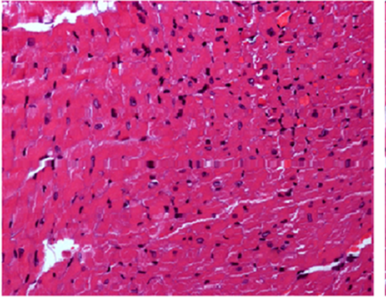

$\mathrm{DM}+$ circHIPK3-shRNA

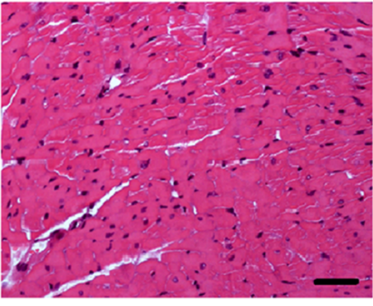

DM + Scr-shRNA was stained with $\mathrm{H} \& \mathrm{E}$ and myocyte cross-sectional area was measured. Fold relative to control. Scale bar, $100 \mu \mathrm{m} . n=5, * p<0.05$. DM, diabetic mice

Fluorescent in situ hybridisation Fluorescent in situ hybridisation (FISH) was employed to detect circHIPK3 RNA and miR-29b-3p expression in cardiac fibroblasts. After prehybridisation, cells were incubated with RNA probes (GenePharma, Suzhou, China) in hybridisation buffer. DNA was stained with DAPI and the signal was measured using an SP5 confocal microscope (Leica Microsystems). The sequences of probes were as follows: circHIPK3, 5'-TGGG TAGACCAAGACTTGTGAGGCCATACCTGTAGTAGC GAGA-3'; miR-29b-3p, 5'-AACACTGATTTCAAATGGTG CTA-3'.

RNA immunoprecipitation assay An RNA immunoprecipitation (RIP) assay, using a Magna RIP Kit (Millipore, Billerica, 
Fig. 3 circHIPK3 knockdown improves cardiac structure and function in diabetic mice. $(\mathbf{a}-\mathbf{c})$ Left ventricular structure was assessed by measurements of LVESD and LVEDD. (d, e) Left ventricular function was evaluated by measurements of LVFS and LVEF. $n=5$, $* p<0.05$. DM, diabetic mice

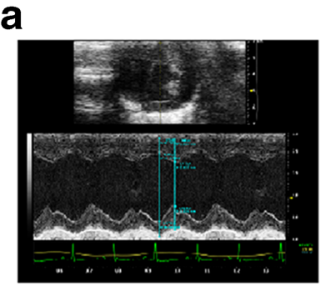

Control

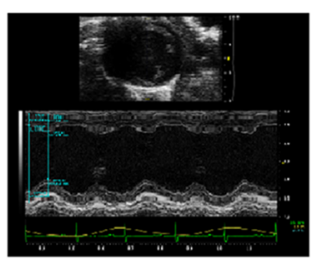

$\mathrm{DM}$

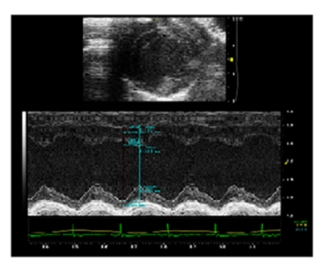

DM + circHIPK3-shRNA

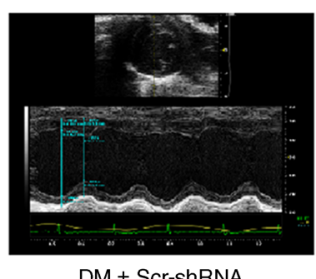

$\mathrm{DM}+$ Scr-shRNA

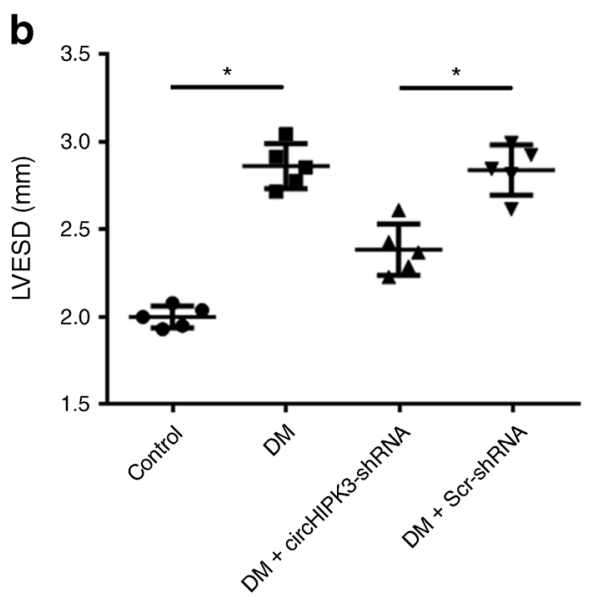

C

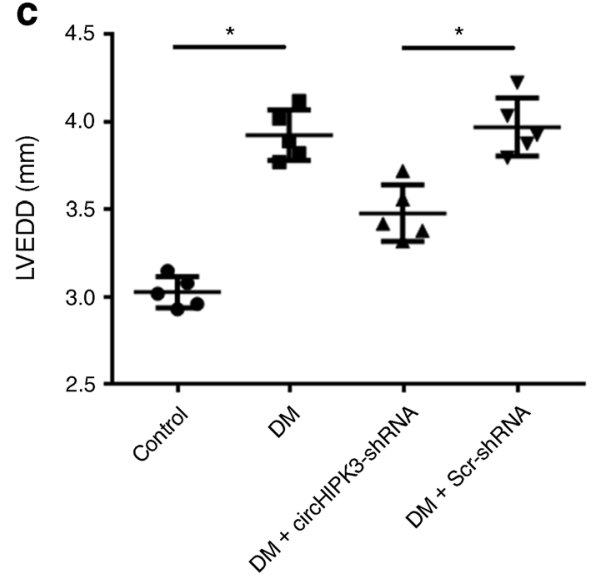

d

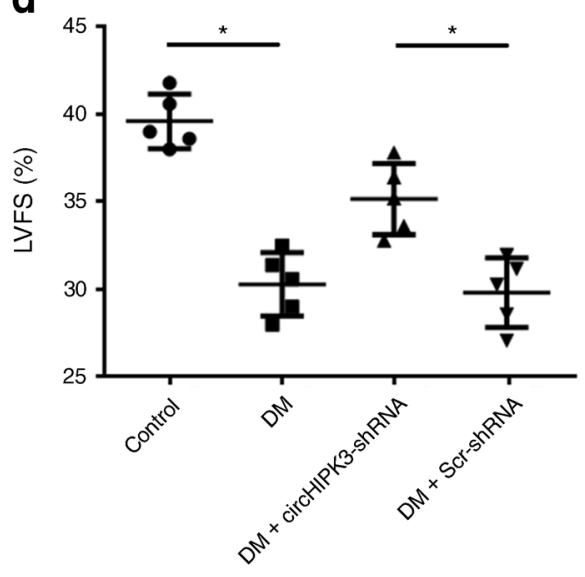

e

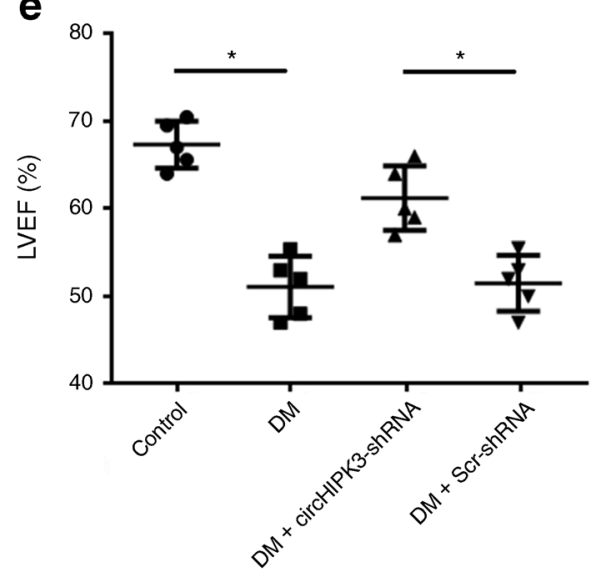

MA, USA), was applied to analyse binding of circHIPK3 and miR-29b-3p to Argonaute 2 (AGO2). Briefly, we lysed cardiac fibroblasts in RIP lysis buffer $(100 \mu \mathrm{l})$ for $10 \mathrm{~min}$. Then, we diluted cell lysates with RIP immunoprecipitation buffer and incubated them with magnetic beads conjugated to antiAGO2 or anti-IgG antibody for $3 \mathrm{~h}$ at $4{ }^{\circ} \mathrm{C}$. Finally, we purified immunoprecipitated RNA and subjected it to PCR.

Luciferase reporter assay Cardiac fibroblasts were seeded onto 96-well plates and incubated overnight. The cells were then co-transfected with Luc-circHIPK3-Wt, Luc-Collal-Wt or Luc-Col3a1-Wt reporter plasmids and their respective mutated vectors (Luc-circHIPK3-Mut, Luc-Colla1-Mut or Luc-
Col3al-Mut) with miR-29b-3p mimic, using Lipofectamine 3000 (Invitrogen, Carlsbad, CA, USA). The dual luciferase reporter assays were conducted to determine the luciferase activity after $48 \mathrm{~h}$ of transfection.

Immunofluorescence staining Cardiac fibroblasts were fixed in $4 \%$ (wt/vol.) paraformaldehyde for $10 \mathrm{~min}$, permeabilised with $0.5 \%$ (vol./vol.) Triton X-100 for $20 \mathrm{~min}$ and incubated with blocking buffer for $30 \mathrm{~min}$ at room temperature. We then incubated fibroblasts overnight at $4^{\circ} \mathrm{C}$ with anti-collagen, type I, alpha 1 (COL1A1) and anti-collagen, type III, alpha 1 (COL3A1) primary antibodies (Abcam). Subsequently, we incubated the cells with Alexa Fluor 488-conjugated 
secondary antibody for $1 \mathrm{~h}$ at room temperature, stained the DNA with DAPI and measured the signal using a confocal laser microscope (Leica Microsystems).

Real-time PCR Total RNAs from cell lysates or myocardial tissue were isolated using TRIzol reagent (Invitrogen). The fractions of nucleus and cytoplasm were separated using NE-PER Nuclear Extraction Reagents (Thermo Scientific, Rockford, USA). Real-time PCR was conducted to quantify the amount of circRNA, miRNA, and mRNA using SYBR Premix Ex Taqkit (TaKaRa, Dalian, China). The $2^{-\Delta \Delta C_{t}}$ method was used to analyse the relative expression of genes. The following primer sequences were used: circHIPK3, forward 5'-GGATCGGCCAGTCATGTATC-3', reverse 5'ACCG CTTGGCTCTACTTTGA-3'; Collal, forward 5'GTGTTCCCTACTCAGCCGTC-3', reverse 5'-GAGG
CCTCGGTGGACATTAG-3'; Col3al, forward 5'-CCCA TGACTGTCCCACGTAA-3', reverse 5'-TTTA CATTGCCACTGGCCTGA-3'; miR-29b-3p, forward 5'GGGTAGCACCATTTGAAATC-3', reverse 5'-CAGT GCGTGTCGTGGAGT-3'，RT-primer， 5'-GTCG TATCCAGTGCGTGTCGTGGAGTCGGCAATTGCAC TGGATACGACAACACT-3'; Gapdh, forward 5'-AAGG TCATCCCAGAGCTGAA-3', reverse 5'-CTGC TTCACCACCTTCTTGA-3'.

Western blotting Cardiac fibroblasts were lysed in an ice-cold lysis buffer and equal amounts of protein were loaded on SDSPAGE for electrophoresis. The blots were probed with rabbit anti-mouse COL1A1 and COL3A1 antibodies (Abcam; 1:1000 in $0.1 \%$ TBST) overnight at $4{ }^{\circ} \mathrm{C}$, and then incubated with horseradish peroxidase-conjugated goat anti-rabbit secondary

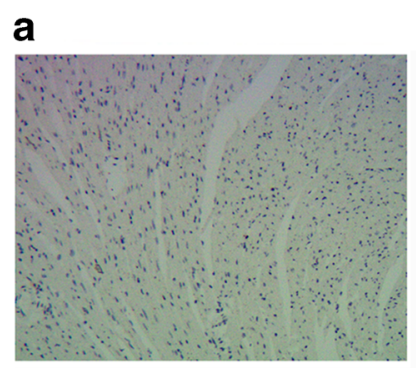

Control

b

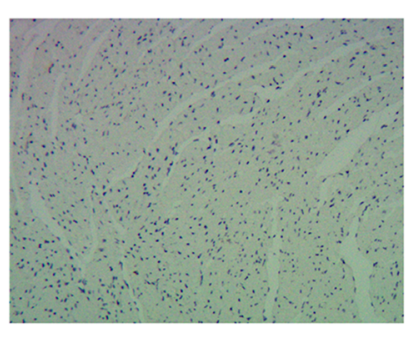

Control
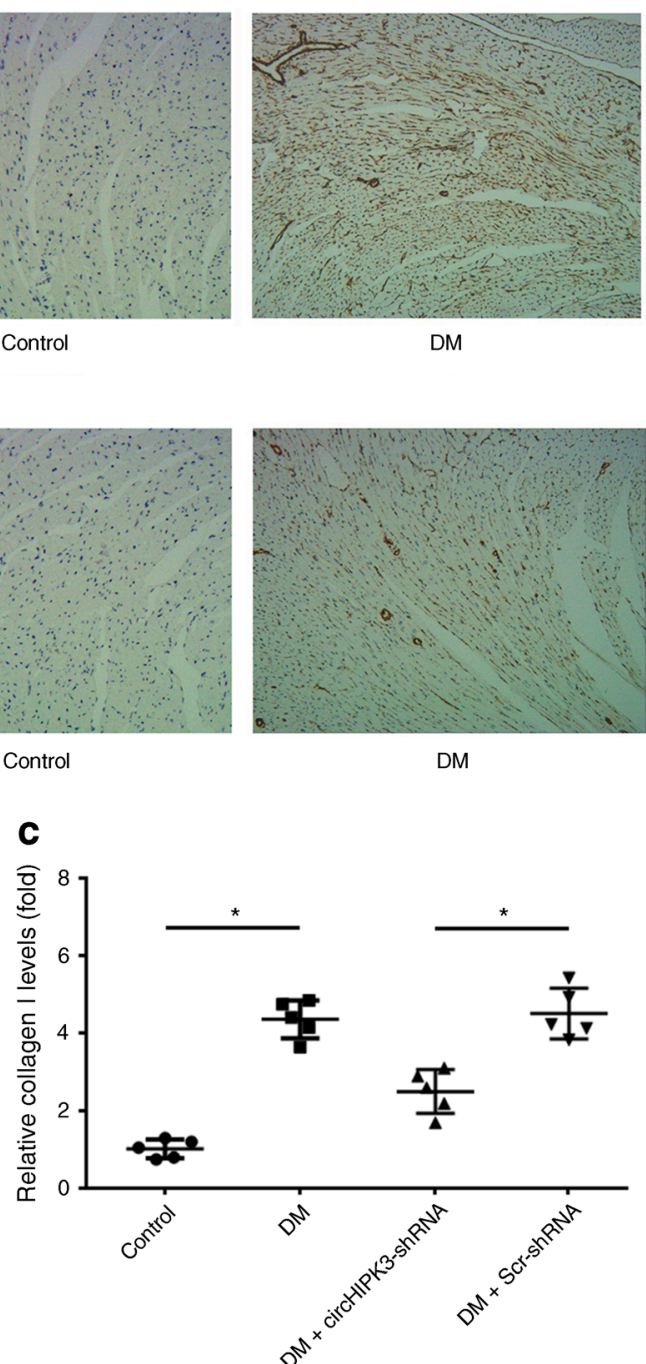

DM

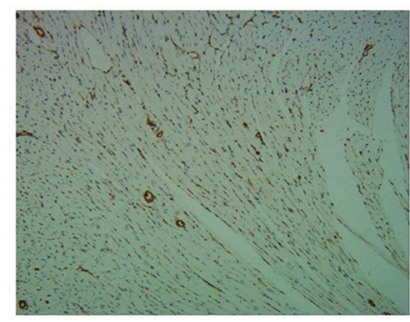

DM

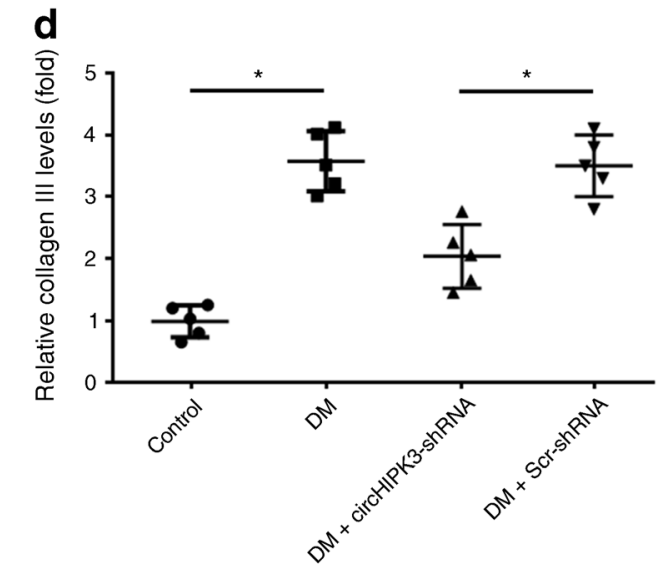

Fig. 4 circHIPK3 is associated with myocardial fibrosis in diabetic mice. Immunohistochemistry was carried out to detect the expression of type I collagen (a, c) and type III collagen $(\mathbf{b}, \mathbf{d})$. Fold relative to control. Scale bar, $100 \mu \mathrm{m} . n=5, * p<0.05$. DM, diabetic mice 
antibody for $1 \mathrm{~h}$ at room temperature. The blots were visualised using an enhanced chemiluminescence kit (Thermo Scientific).

Statistical analysis Statistical analysis was carried out using SPSS version 20.0 (IBM, Chicago, IL, USA). The experimental data were expressed as mean $\pm \mathrm{SD}$. The differences between groups were compared using Student's $t$ test or one-way ANOVA. A $p$ value of $<0.05$ was considered statistically significant.

Fig. 5 circHIPK3 is involved in the regulation of cardiac fibroblast proliferation. (a) The FISH assay was used to detect circHIPK3 expression in mouse cardiac fibroblasts. (b) The cardiac fibroblasts were transfected with adenovirus containing circHIPK3-siRNA or Scr-siRNA and exposed to Ang II $(1 \mu \mathrm{mol} / \mathrm{l})$ for $48 \mathrm{~h}$. The expression of circHIPK3 was examined by real-time PCR. (c, d) The proliferation of cardiac fibroblasts was measured by the EdU incorporation assay. Fold relative to control. Scale bar, $100 \mu \mathrm{m} . n=5, * p<0.05$

a

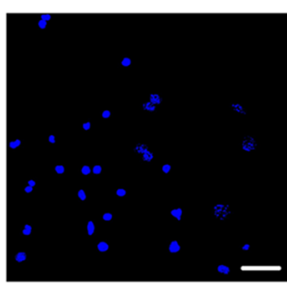

DAPI

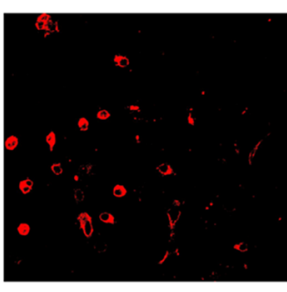

circHIPK3

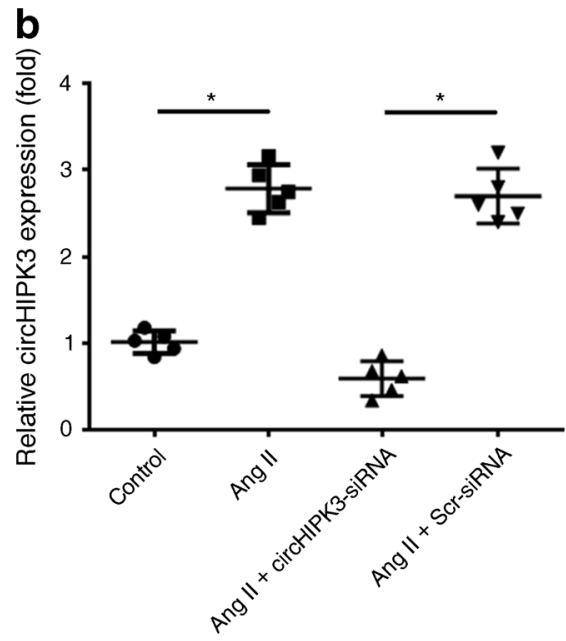

d
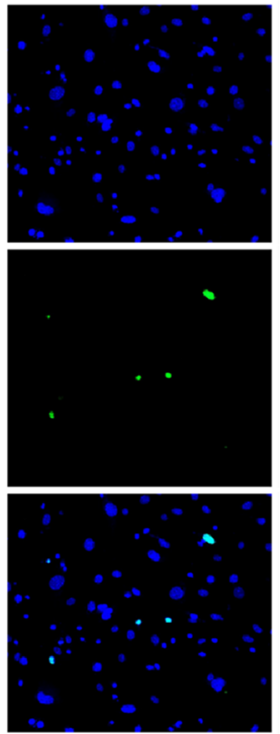

Control
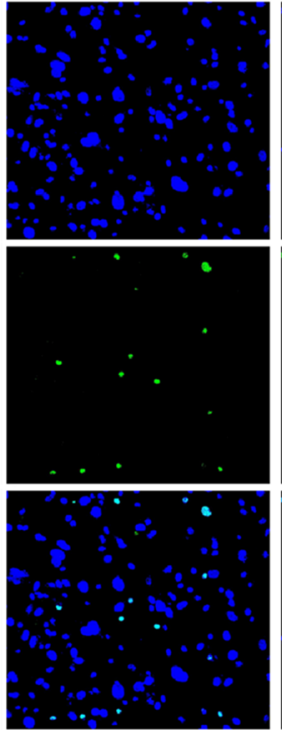

Ang II

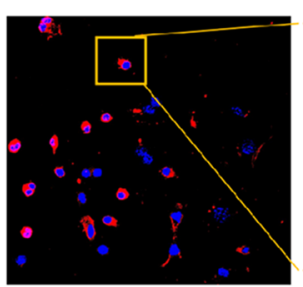

Merge

C

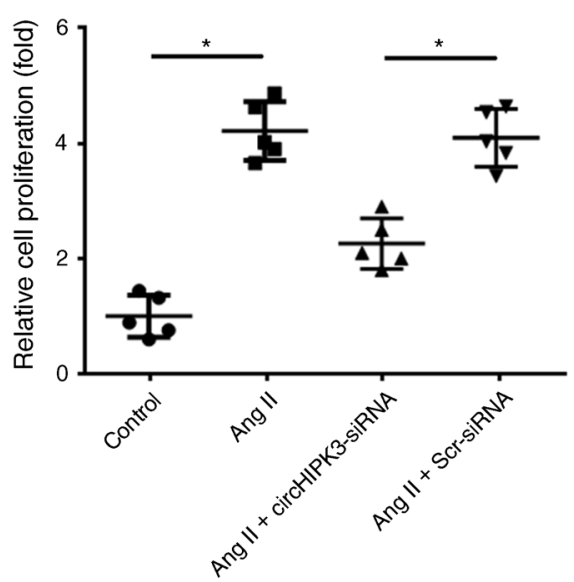

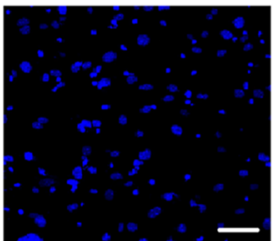

Hochest
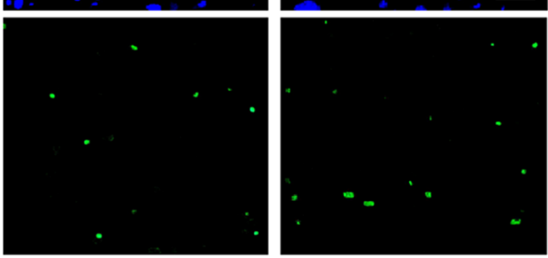

Alex fluro 488

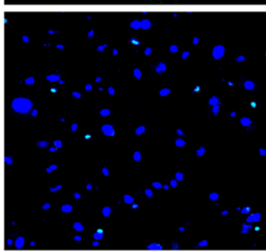

Ang II+circHIPK3-siRNA

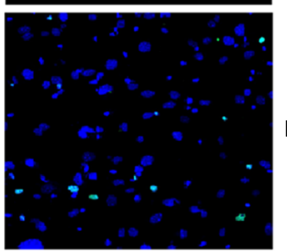

Ang II+Scr-siRNA 
Fig. 6 circHIPK3 is targeted by miR-29b-3p in cardiac

fibroblasts. (a) Cardiac fibroblasts were co-transfected with miRNA mimics and Luc-circHIPK3-Wt or Luc-circHIPK3-Mut, and $48 \mathrm{~h}$ later, a dual luciferase reporter assay was carried out (fold relative to control; $n=3$, $* p<0.05$ ). (b) The RIP assay was applied to detect the binding of circHIPK3 and miR-29b-3p with AGO2 (fold relative to anti$\mathrm{AGO} 2 ; n=5, * p<0.05)$. (c) Bioinformatics analysis indicated that circHIPK3 sequence contains the binding site for miR-29b-3p. (d) The FISH assay was performed to determine the colocalisation of circHIPK 3 and miR-29b-3p in the cytoplasm of cardiac fibroblasts. Scale bar, $100 \mu \mathrm{m}$
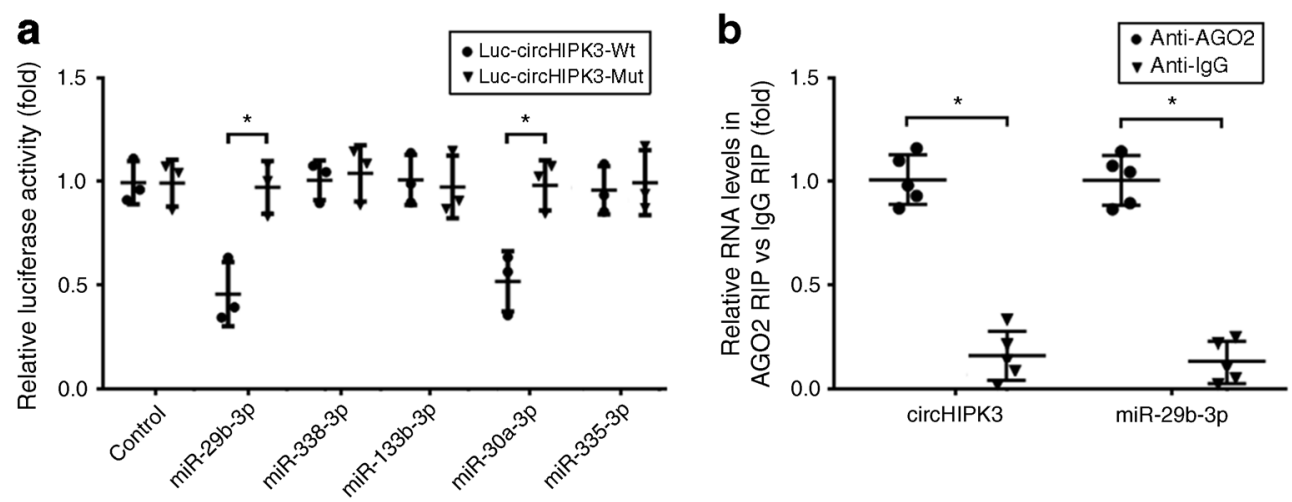

C

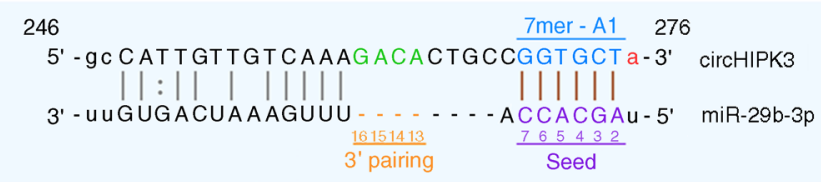

d
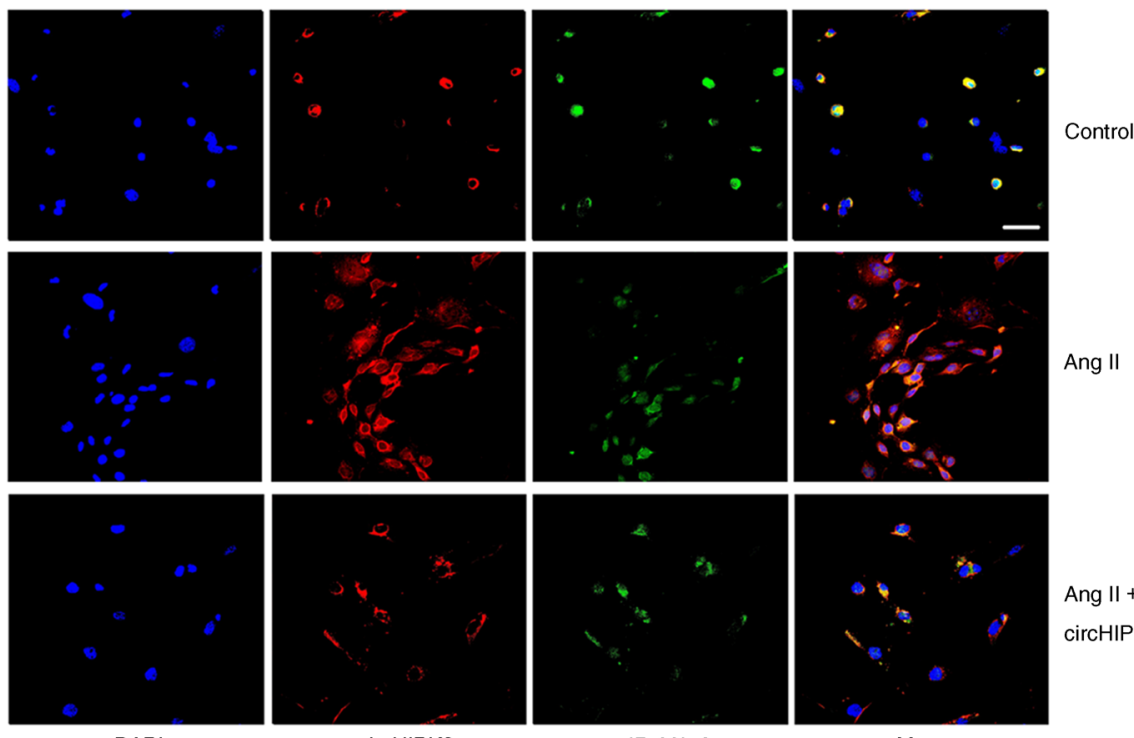

miR-29b-3p

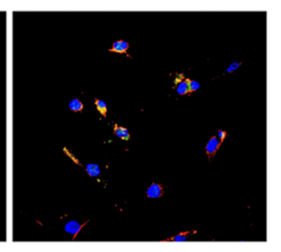

Ang II +

circHIPK3-siRNA

circHIPK3

Merge was resistant to RNase R digestion, while Hipk3 mRNA was easily degraded (Fig. 1b). The results of PCR showed that circHIPK3 was mainly expressed in the cytoplasm of cardiac fibroblasts (Fig. 1c). Furthermore, a high glucose concentration was found to upregulate circHIPK3 expression in cardiac fibroblasts (Fig. 1d). Gel electrophoresis confirmed the PCR product of circHIPK3 and Sanger sequencing verified the head-to-tail splicing of the product (Fig. 1e).

\section{circHIPK3 silencing reduces histological abnormalities in} diabetic mice As shown in Fig. 2a, circHIPK3 expression was increased in the myocardium of diabetic mice and decreased after transfection with circHIPK3-shRNA.
Cardiac tissue was stained with Masson and H\&E, and collagen area fraction and myocyte cross-sectional area were determined. Masson staining showed that circHIPK3 knockdown inhibited myocardial fibrosis in diabetic mice (Fig. 2b, d). H\&E staining indicated that circHIPK3 silencing attenuated cardiac hypertrophy in diabetic mice (Fig. 2c, e).

circHIPK3 knockdown improves cardiac structure and function in diabetic mice Echocardiographic measurements were used to evaluate cardiac structure and function. LVESD and LVEDD were elevated in diabetic mice and reduced in diabetic mice transfected with circHIPK3-shRNA (Fig. 3a-c). In 
Fig. 7 Collal and Col3al are downstream targets of miR-29b3p. (a, b) Bioinformatics analysis showed that Collal and Col3al sequences contain the binding sites for miR-29b-3p. (c, d) The luciferase assay was conducted to confirm the binding of miR-29b$3 p$ to Colla1 and Col3a1 (fold relative to miR-NC). (e) Mouse cardiac fibroblasts were transfected with adenovirus circHIPK3 and/or miR-29b-3p mimic for $48 \mathrm{~h}$ and then western blot analysis was used to determine the expression of COL1A1 and COL3A1 (fold relative to control). (f) The luciferase assay indicated that circHIPK3 could competitively inhibit the binding of miR-29b-3p to Collal and Col3al. miR-NC, miRNA negative control. $n=5$, $* p<0.05$ a

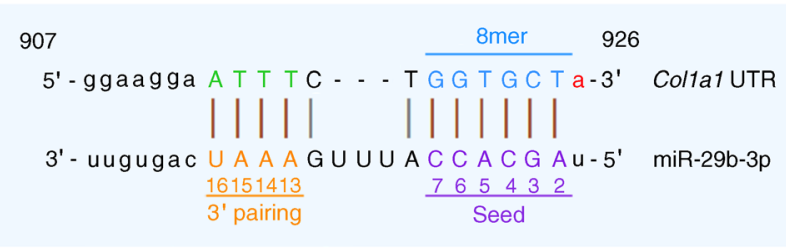

b
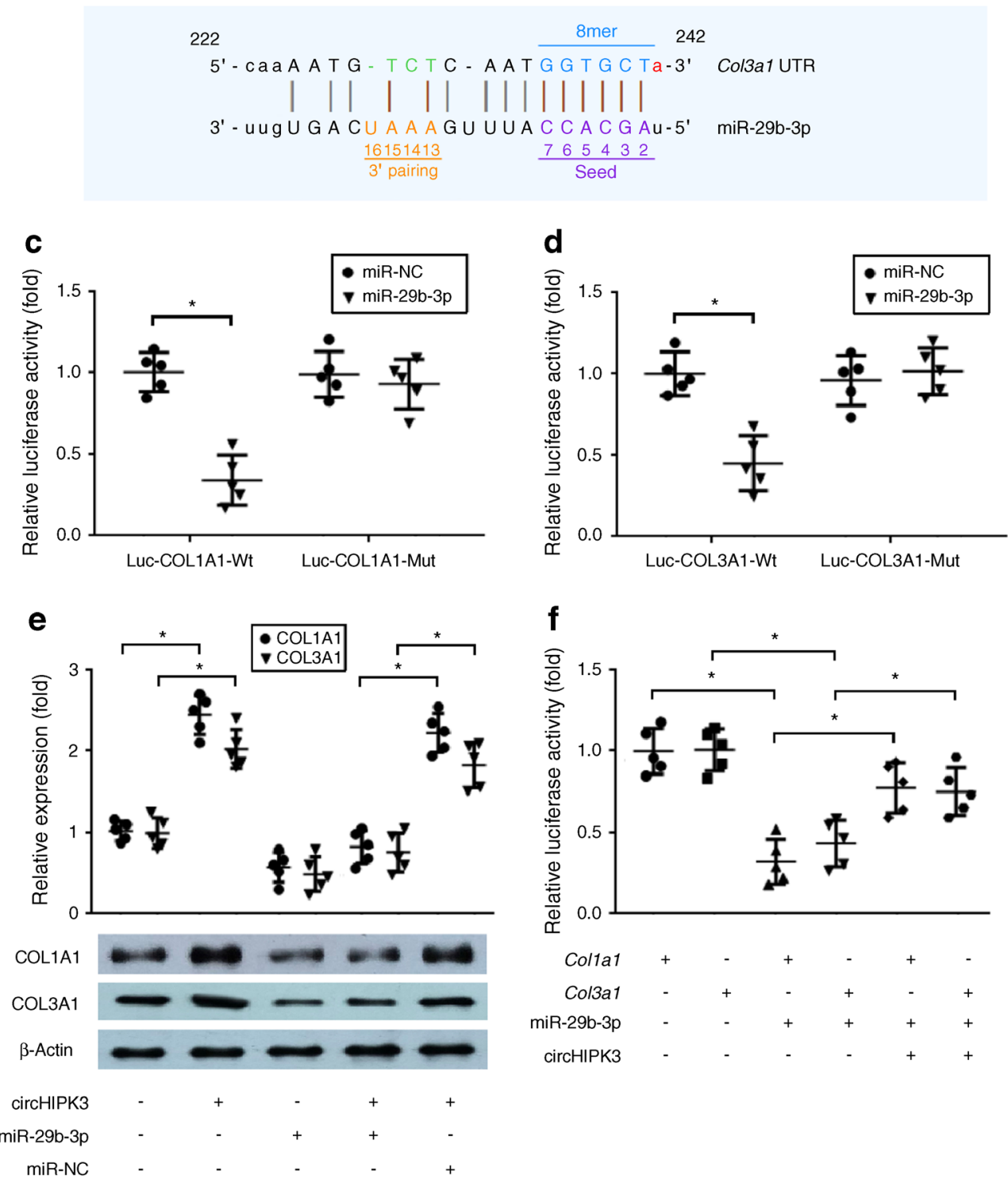

addition, LVFS and LVEF were decreased in diabetic mice, while circHIPK3 silencing was found to improve left ventricular systolic function in diabetic mice (Fig. 3d, e).

circHIPK3 is associated with myocardial fibrosis in diabetic mice Immunohistochemistry was employed to detect the expression of fibrosis-related proteins in myocardial tissue. The expression of types I and III collagen was significantly elevated in the diabetic myocardium, while circHIPK3 silencing was found to decrease the expression of fibrosis-associated proteins (Fig. 4a-d).

circHIPK3 is involved in the regulation of cardiac fibroblast proliferation The FISH assay showed that circHIPK3 is primarily expressed in the cytoplasm of cardiac fibroblasts (Fig. 5a). circHIPK3 expression was upregulated 


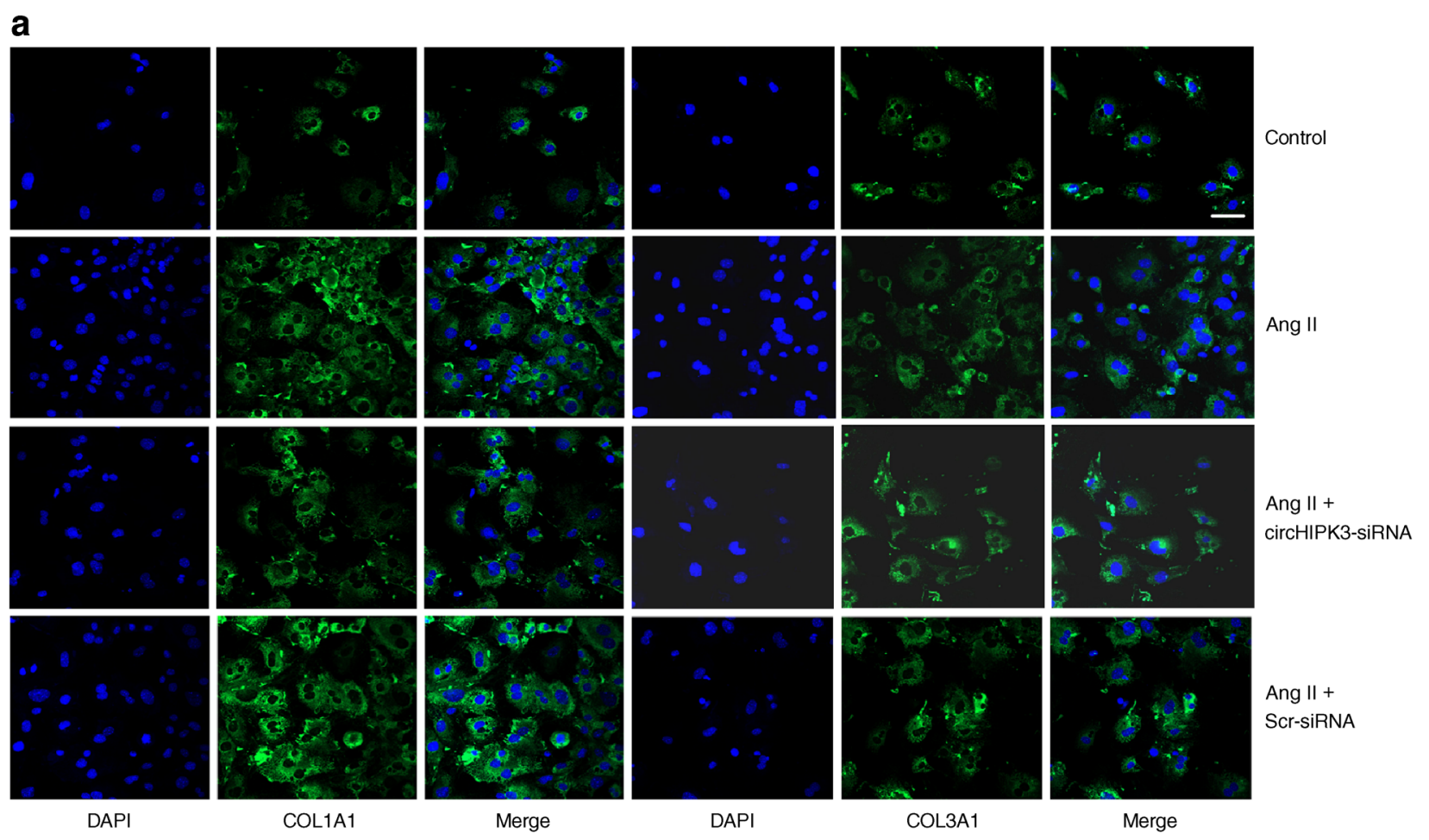

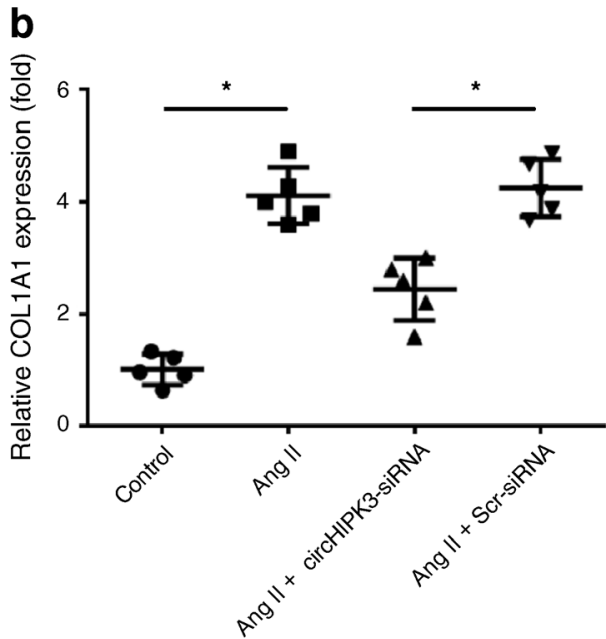

Fig. 8 Ang II promotes the expression of COL1A1 and COL3A1 via circHIPK3. (a-c) Mouse cardiac fibroblasts were transfected with adenovirus containing circHIPK3-siRNA or Scr-siRNA and then exposed to

in cardiac fibroblasts treated with Ang II (Fig. 5b). The EdU incorporation assay revealed that Ang II treatment was associated with increased proliferation of cardiac fibroblasts. Knockdown of circHIPK3, on the other hand, was found to suppress Ang II-induced cell proliferation (Fig. 5c, d).

circHIPK3 is targeted by miR-29b-3p in cardiac fibroblasts Bioinformatics prediction showed that circHIPK3 sequence contains the binding sites for miR-29b-3p, miR-338-3p, miR133b-3p, miR-30a-3p and miR-335-3p. Cardiac fibroblasts were

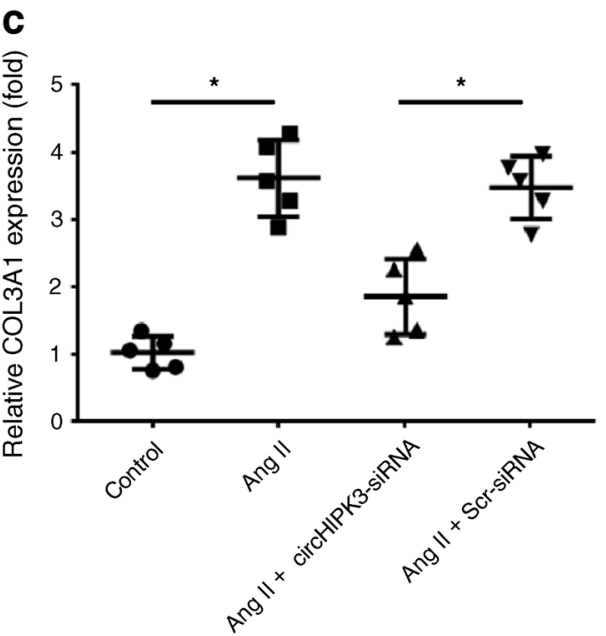

Ang II ( $1 \mu \mathrm{mol} / \mathrm{l})$ for $48 \mathrm{~h}$. Immunofluorescence staining was performed to detect the expression of COL1A1 and COL3A1. Fold relative to control. Scale bar, $100 \mu \mathrm{m} . n=5, * p<0.05$

co-transfected with miRNA mimics and Luc-circHIPK3-Wt or Luc-circHIPK3-Mut, and dual luciferase reporter assays were carried out after $48 \mathrm{~h}$. The results showed that miR-29b-3p reduced the luciferase activity of Luc-circHIPK3-Wt but did not affect the luciferase activity of Luc-circHIPK3-Mut (Fig. 6a). The RIP assay indicated that miR-29b-3p and circHIPK3 were specifically enriched in the immunoprecipitate pulled-down by an antibody against AGO2, a core component of the RNAinduced silencing complex (Fig. 6b). The target binding sites of miR-29b-3p in circHIPK3 are illustrated in Fig. 6c. The FISH assay suggested that miR-29b-3p and circHIPK 3 co-localised in 
the cytoplasm of cardiac fibroblasts and that co-localisation of these two molecules increased following treatment with Ang II (Fig. 6d).

\section{Col1a1 and Col3a1 are downstream targets of miR-29b-3p} Bioinformatics analysis confirmed that Collal and Col3al sequences contain the binding sites of miR-29b-3p (Fig. $7 \mathrm{a}, \mathrm{b})$. The 3'-untranslated regions of Collal and Col3Al were fused to the luciferase coding region and transfected into cardiac fibroblasts with miR-29b-3p mimic. The luciferase assay indicated that Collal and Col3al were the direct targets of miR-29b-3p (Fig. 7c, d). The expression of COL1A1 and COL3A1 was upregulated in cardiac fibroblasts transfected with adenovirus containing circHIPK3, while their expression was downregulated when fibroblasts were co-transfected with circHIPK3 and miR-29b-3p mimic (Fig. 7e). Additionally, overexpression of circHIPK3 counteracted the inhibitory effects of miR-29b-3p on COL1A1 and COL3A1 (Fig. 7f).

\section{Ang II promotes the expression of COL1A1 and COL3A1 in mouse cardiac fibroblasts via circHIPK3 Immunofluorescence staining showed that Ang II was associated with increased expression of COL1A1 and COL3A1, which are important components of types I and III collagen. However, silencing of circHIPK3 was found to downregulate COL1A1 and COL3A1 expression, which consequently attenuates myocar- dial fibrosis (Fig. 8a-c).}

\section{Discussion}

Accumulating evidence suggests that circRNAs are essential in the pathogenesis of various CVDs [6, 7]. However, we know less about the pathological role of circRNAs in DCM. In the current study, we found circHIPK 3 to be significantly upregulated in the myocardium of diabetic mice. We therefore performed in vitro and in vivo experiments to investigate the molecular mechanisms linking circHIPK3 to the development of DCM. Our results reveal that knockdown of circHIPK 3 can reduce myocardial fibrosis and improve left ventricular function in a mouse model of DCM. CircHIPK3 may function as a competing endogenous RNA (ceRNA) that upregulates COL1A1 and COL3A1 expression by suppressing miR-29b$3 \mathrm{p}$, which consequently contributes to increased cardiac fibrosis and progression of DCM.

Many studies have demonstrated that circHIPK3 exerts a variety of biological effects by inhibiting different miRNAs. circHIPK 3 participates in the development of diabetic retinopathy by blocking the function of miR-30a, resulting in increased vascular dysfunction and endothelial proliferation [8]. circHIPK3 also enhances the growth and metastasis of colorectal cancer by suppressing miR-7 and may function as a prognostic biomarker in colorectal cancer [9]. Additionally, circHIPK 3 regulates cell migration and proliferation by targeting miR-124, thereby increasing aquaporin 3 expression in hepatocellular carcinoma [10]. In the current study, we discovered a new regulatory network, circHIPK3-miR-29b$3 \mathrm{p}-$ Colla1-Col3al, which is crucially involved in the pathogenesis of DCM.

Cardiac fibrosis is defined as an imbalance between production and degradation of extracellular matrix, which contributes to the accumulation of connective tissue protein in the perivascular and interstitial tissues and has an essential function in the development of cardiac remodelling and heart failure. Accumulating evidence has revealed that cardiac fibrosis is actively involved in the pathological processes underlying DCM $[1,2]$. Development of myocardial fibrosis in DCM involves the deposition and crosslinking of stiff collagen, perivascular fibrosis, cardiac interstitial fibrosis, progressive abolition of muscular fibrils and thickening of the basement membrane [11, 12]. Many proteins participate in the pathological process of myocardial fibrosis, with COL1A1 and COL3A1 being of particular importance. Our data show that circHIPK3 could promote myocardial fibrosis in DCM by upregulating the expression of Collal and Col3al, thus increasing the synthesis of types I and III collagen. The ceRNA regulatory network, circHIPK3-miR-29b-3p-Col1a1-Col3a1, will provide novel insights into the pathogenesis and treatment of DCM.

In conclusion, circHIPK3 is upregulated in the diabetic myocardium and it may function as a ceRNA that increases Collal and Col3al expression by inhibiting miR-29b-3p, thus leading to myocardial fibrosis in DCM.

Supplementary Information The online version contains peer-reviewed but unedited supplementary material available at https://doi.org/10.1007/ s00125-020-05353-8.

Data availability The datasets generated during the current study are available from the corresponding author upon reasonable request.

Funding This study was financially supported by the National Natural Science Foundation of China (81700737), the Scientific Research Program for Young Talents of China National Nuclear Corporation (51001), the Gusu Health Talents Training Project (GSWS2019045) and the Talent Support Project of the Second Affiliated Hospital of Soochow University (XKTJ-RC202003).

Authors' relationships and activities The authors declare that there are no relationships or activities that might bias, or be perceived to bias, their work.

Contribution statement All authors provided substantial contributions to study conception and design, acquisition of data, or analysis and interpretation of data, drafting the article and revising it critically for important intellectual content, and gave final approval of the version to be published. XZ designed the study; WW and SZ drafted the manuscript; $\mathrm{XZ}$ is responsible for the integrity of the work as a whole. 


\section{References}

1. Jia G, Hill MA, Sowers JR (2018) Diabetic cardiomyopathy: an update of mechanisms contributing to this clinical entity. Circ Res 122:624-638. https://doi.org/10.1161/CIRCRESAHA.117.311586

2. Marwick TH, Ritchie R, Shaw JE, Kaye D (2018) Implications of underlying mechanisms for the recognition and management of diabetic cardiomyopathy. J Am Coll Cardiol 71:339-351

3. Park S, Nguyen NB, Pezhouman A, Ardehali R (2019) Cardiac fibrosis: potential therapeutic targets. Transl Res 209:121-137. https://doi.org/10.1016/j.trsl.2019.03.001

4. Qu S, Yang X, Li X et al (2015) Circular RNA: a new star of noncoding RNAs. Cancer Lett 365:141-148. https://doi.org/10. 1016/j.canlet.2015.06.003

5. Chen LL (2016) The biogenesis and emerging roles of circular RNAs. Nat Rev Mol Cell Biol 17:205-211. https://doi.org/10. 1038/nrm.2015.32

6. Zhou MY, Yang JM, Xiong XD (2018) The emerging landscape of circular RNA in cardiovascular diseases. J Mol Cell Cardiol 122: 134-139. https://doi.org/10.1016/j.yjmcc.2018.08.012

7. Gomes CPC, Salgado-Somoza A, Creemers EE, Dieterich C, Lustrek M, Devaux Y (2018) Circular RNAs in the cardiovascular system. Noncoding RNA Res 3:1-11
8. Shan K, Liu C, Liu BH et al (2017) Circular noncoding RNA HIPK3 mediates retinal vascular dysfunction in diabetes mellitus. Circulation. 136:1629-1642. https://doi.org/10.1161/ CIRCULATIONAHA.117.029004

9. Zeng K, Chen X, Xu M et al (2018) CircHIPK3 promotes colorectal cancer growth and metastasis by sponging miR-7. Cell Death Dis 9: 417

10. Chen G, Shi Y, Liu M, Sun J (2018) circHIPK3 regulates cell proliferation and migration by sponging miR-124 and regulating AQP3 expression in hepatocellular carcinoma. Cell Death Dis 9: 175

11. Asbun J, Villarreal FJ (2006) The pathogenesis of myocardial fibrosis in the setting of diabetic cardiomyopathy. J Am Coll Cardiol 47: 693-700

12. Russo I, Frangogiannis NG (2016) Diabetes-associated cardiac fibrosis: cellular effectors, molecular mechanisms and therapeutic opportunities. J Mol Cell Cardiol 90:84-93. https://doi.org/10. 1016/j.yjmcc.2015.12.011

Publisher's note Springer Nature remains neutral with regard to jurisdictional claims in published maps and institutional affiliations. 